\title{
FORUM ON
}

IMMUNOPATHOLOGICAL

\section{DISEASES AND THERAPEUTICS}

CONTENTS VOLUME 6, 2015

Page Range of Issues; Issues 1-2: 1-131; Issues 3-4: 133-261

ISSUES 1-2

JOHN L. FAHEY: PIONEeR In NeW DisCiPlines OF HUMAN IMMUNOlOGY GUEST EditorS: OTONIEL MARTínEZ-MAZA \& CHRISTEL H. UITTENBOGAART

Eulogy to Professor John L. Fahey: My Teacher, Collaborator, and Professional Counselor B. Bonavida

John L. Fahey: A Man of Many Talents J. Zighelboim

Reinvigorating Exhausted T Cells by Blockade of the PD-1 Pathway J. Lee, E. Ahn, H.T. Kissick, \& R. Ahmed

Measurement of Circulating Cytokines and Immune-Activation Markers by Multiplex Technology in the Clinical Setting: What Are We Really Measuring? N. Aziz

Lessons of Life and Science from Dr. John L. Fahey S.F. Plaeger

Memories of John L. Fahey, M.D.'s Clinical Immunology Society Years

S. Kanowith-Klein

Worms and Humans: A Happy Divorce? H. Akuffo, S. Britton, \& T. Schön

Human T-Cell Development and Thymic Egress: An Infectious Disease Perspective R.S. Resop \& C.H. Uittenbogaart

Fond Memories of John Fahey R. Detels

Memories of John Fahey and His Contributions to the Multicenter AIDS Cohort Study (MACS) J. Phair, R. Detels, J. Margolick, C. Rinaldo, L. Jacobson, O. Martínez-Maza, S. Wolinsky, \& A. Muñoz

A Brief Chronicle of CD4 as a Biomarker for HIV/AIDS: A Tribute to the Memory of John L. Fahey J.M. Kagan, A.M. Sanchez, A. Landay, \& T.N. Denny

Programming T Cell Killers for an HIV Cure: Teach the New Dogs New Tricks and Let the Sleeping Dogs Lie K.N. Smith, R.B. Mailliard, \& C.R. Rinaldo

Immune Activation: Contribution to AIDS-Associated Non-Hodgkin Lymphoma M. Epeldegui \& O. Martínez-Maza

Experimental Approaches for Eliminating Latent HIV M.D. Marsden \& J.A. Zack

Development of Indian HIV-1 Subtype C Vaccine Candidates P. Seth \& S.S. Yadav 
The Legacy of John L. Fahey in Psychoneuroimmunology Research E.C. Breen

Translational Psychoneuroimmunology in Oral Biology and Medicine F. Chiappelli, A. Barkhordarian, Q. Bach, \& G. Demerjian

\section{ISSUES 3-4}

\section{MiCROVESICLES IN HUMAN DISEASES AND THEIR ROLE IN INTERCELLULAR COMMUNICATION AND SIGNALING GUEST EDITORS: FEDERICA VANNINI \& FRANCESCO RUSSO}

Extracellular Vesicles as Potential Mediators of Epigenetic Reprogramming A.L. Ronnegren

Tissue Cross-Talk and Exosomal MicroRNAs M. Marchetti

Extracellular Vesicle-Mediated Transfer of MicroRNAs in Atherosclerosis F. Vannini \& F. Russo

Extracellular Vesicles: Evolving Contributors in Autoimmunity S. Katsiougiannis

Exosomes, Ectosomes and the Two Together. Physiology and Pathology J. Meldolesi

Circular RNAs and Exosomes: The New Frontier of Cancer Diagnosis F. Scoyni \& R. Giugno

MANIPULATION OF MACROPHAGE FUNCTIONS BY MYCOBACTERIUM TUBERCULOSIS GUEST EDITOR: MAGDALENA KLINK

Phosphorylation Signaling in Mycobacterium Tuberculosis: An Overview A.C.O. Menegatti \& H. Terenzi

Modulations of Macrophage Immune Responses by Mycobacterial PE/PPE Family of Proteins S. Nair

Autophagy and Autophagy-Related Proteins in the Immunity against Mycobacterium Tuberculosis A. Bah \& I. Vergne

Toll Like Receptor-2 Signaling in Mycobacterium Tuberculosis Infection-A Double-Edged Sword M. Sharma \& S. Sharma

The Role of Homologous Recombination and Non-Homologous Ends Joining Systems in $M$. Tuberculosis Survival inside Macrophages I. Szulc-Kielbik, M. Kielbik, \& M. Klink

Role of Dendritic Cells in Host-Mycobacterium Bovis BCG Interactions 


\section{FORUM ON}

\section{IMMUNOPATHOLOGICAL DISEASES AND THERAPEUTICS}

AUTHOR INDEX, Volume 6, 2015

Page Range of Issues; Issue 1-2: 1-131; Issue 3-4: 133-261

A.M. Sanchez, 57

Ahmed, R., 7

Ahn, E., 7

Akuffo, H., 27

Aziz, N., 19

Bach, Q., 119

Bah, A., 217

Barkhordarian, A., 119

Bonavida, B., 1

Breen, E.C., 109

Britton, S., 27

Chiappelli, F., 119

Demerjian, G., 119

Denny, T.N., 57

Detels, R., 51, 53

Giugno, R., 181

Epeldegui, M., 79

Jacobson, L., 53

Kagan, J.M., 57

Kanowith-Klein, S., 25

Katsiougiannis, S., 163

Kielbik, M., 237

Kissick, H.T., 7

Klink, M., 237

Kowalewicz-Kulbat, M., 251

Krawczyk, K., 251

Landay, A., 57

Lee, J., 7

Mailliard, R.B., 67

Marchetti, M., 143
Margolick, J., 53

Marsden, M.D., 91

Martínez-Maza, O., 53, 79

Meldolesi, J., 171

Menegatti, A.C.O., 191

Muñoz, A., 53

Nair, S., 207

Phair, J., 53

Plaeger, S.F., 23

Resop, R.S., 33

Rinaldo, C.R., 53, 67

Ronnegren, A.L., 133

Rudnicka, W., 251

Russo, F., 157

Schön, T., 27

Scoyni, F., 181

Seth, P., 101

Sharma, M., 227

Sharma, S., 227

Smith, K.N., 67

Szulc-Kielbik, I., 237

Terenzi, H., 191

Uittenbogaart, C.H., 33

Vannini, F., 157

Vergne, I., 217

Wolinsky, S., 53

Yadav, S.S., 101

Zack, J.A., 91

Zighelboim, J., 3 


\title{
FORUM ON
}

\section{IMMUNOPATHOLOGICAL DISEASES AND THERAPEUTICS}

SubJeCt INDEX, Volume 6, 2015

\author{
Page Range of Issues; Issue 1-2: 1-131; Issue 3-4: 133-261
}

acquired immunodeficiency syndrome (AIDS), 51, 53, 57, 79

activation, 91 apoptotic bodies, 163

atherosclerosis, 157

autoimmunity, 27, 163

autophagy, 217

biomarkers, 57, 143, 157, 181

blood-brain barrier, 119

cancer, 7, 133, 181

cellular communication, 133

chronic infection, 7

circular RNAs, 181

Clinical Immunology Society, 25

confection, 27

crosstalk, 143

cytokine, 19

cytotoxic T cell (CTL), 67

dendritic cells, 67, 251

deoxyribo nucleic acid (DNA), 101

deworming, 27

DNA methylation, 133

DNA vaccine, 101

epigenetic, 133

evidence-based health care, 119

exosomes, 133, 143, 157, 163, 181

extracellular vesicles, 133, 163, 171

flow cytometry, 57

helminths/worms, 27

heterogeneity, 171

histone modification, 133

HIV vaccine, 101

HIV-1 subtype C, 101

homologous recombination sys-

tems, 237

horizontal transfer of cargoes, 171 human immunodeficiency virus

(HIV), 33, 57, 79

human immunodeficiency virus type-1 (HIV-1), 67, 101

human immunology, 33

humanized mice, 33

immune activation, 79

immune monitoring, 57

immune system, 109

immunity, 163, 217

immunology, 19, 51, 53

immunomodulation, 207

immunotherapy, 7, 67

intervention, 109

kinase, 191

latency, 91

luminal cargoes, 171

lymphoma, 79

lysosomes, 217

M. bovis Bacille Calmette-Guerin, 251

macrophage immune responses, 207

macrophages, 217, 237

markers, 171

microRNA, 133, 143, 157

microvesicles, 133, 163

migration, 33

mind-body interactions, 109

multiplex assay, 19

multivesicular bodies, 171

murine models, 33

Mycobacterium tuberculosis, 181, 207, 227, 237

natural history, 53

neuroinflammation, 119 noncoding RNAs, 181

non-Hodgkin lymphoma (NHL), 79

non-homologous end joining sys-

tems, 237

outside/inside membrane fusion, 171

pathogenesis, 207

PE/PPE proteins, 207

phagosome, 217

phosphatase, 191

plasma membrane pinch-off, 171

postdoctoral fellows, 23

preclinical immunogenicity, 101

prevention-tools, 143

prime-boost regimen, 101

programmed cell death-1, 7

psychoneuroimmunology, 109, 119

reservoir, 91

signal transduction, 191

sphingosine-1-phosphate, 33

T-cell exhaustion, 7

T-cell reconstitution, 33

temporomandibular joint disorders, 119

Th1 immune response, 227

Th2 immune response, 227

thymic egress, 33

thymocyte development, 33

thymus, 33

Toll-like receptors, 227

translational science, 119

Tregs, 119

tuberculosis, 27, 217

tyrosine phosphorylation, 191

vaccine, 251

vesicle budding/rolling, 171

vesicle diagnosis, 171

vesicle therapy, 171 